\title{
First report of Neofusicoccum parvum associated with shoot cankers of peach (Prunus persica) in Shanghai, China
}

\author{
Liqing Zhang ${ }^{1} \cdot$ Xiongwei $\mathrm{Li}^{1}$ (D) $\cdot$ Mingshen $\mathrm{Su}^{1} \cdot$ Jingyi Zhou ${ }^{2} \cdot$ Zhengwen $\mathrm{Ye}^{1}$ \\ Received: 12 March 2019 / Accepted: 30 April 2019/Published online: 24 May 2019 \\ (C) Società Italiana di Patologia Vegetale (S.I.Pa.V.) 2019
}

Keywords Peach $\cdot$ Gummosis $\cdot$ Shoot cankers $\cdot$ Neofusicoccum parvum

China is the leading producer of peaches (Prunus persica $\mathrm{L}$. Batsch), providing more than $50 \%$ of the world production. In June 2017, symptoms of gummosis were observed on 3year-old peach trees in the germplasm of Shanghai Academy of Agricultural Sciences, Shanghai, China. Isolation by transferring little tissue samples approximately $(5 \mathrm{~mm} \times 5 \mathrm{~mm})$ of symptomatic branches onto Petri plates containing potato dextrose agar (PDA) yielded colonies with aerial mycelia. Colonies of fungal isolates on PDA showed white aerial mycelium at the early stage and turned gray to dark gray after 7 days. Conidia were hyaline, unicellular, thin-walled with smooth surface, and ellipsoid with round apices, and measured 10.2 to $24.2 \mu \mathrm{m} \times 2.4$ to $8.2 \mu \mathrm{m}$. To confirm the pathogen identity, the internal transcribed spacer (ITS) region and the translation elongation factor 1- $\alpha$ gene $(E F 1-\alpha)$ were amplified from DNA extracted from single-spore cultures of a representative isolate SH01 using primers ITS1/ITS4 (White et al. 1990) and EF1-728F/EF1-986R (Carbone and Kohn 1999). The consensus sequences of ITS (MH512906.1) and EF1- $\alpha$ (MH551174.1) showed 99\% identity with $N$. parvum (KU997467.1 and KU997305.1 for ITS and EF1- $\alpha$, respectively). Pathogenicity tests was conducted by spraying the isolate SH01 on the current year semi-lignified shoots of 4year-old peach trees (cv. 'Hu 277') in Zhuanghang, Shanghai. Gum exudations appeared after 45 days on inoculated regions. No lesions and gum exudations were observed on the control branches. To our knowledge, this is the first report of presence of $N$. parvum associated with canker on peach tree in Shanghai, China.

Funding This study was supported by the National Science Foundation of China (grant no. 31401833).

\section{References}

Carbone I, Kohn LM (1999) A method for designing primer sets for speciation studies in filamentous ascomycetes.[J]. Mycologia 91(3):553-556

White TJ et al (1990) PCR protocols: a guide to methods and applications. Academic Press, San Diego, p 315

Publisher's note Springer Nature remains neutral with regard to jurisdictional claims in published maps and institutional affiliations.
Liqing Zhang and Xiongwei Li contributed equally to this work.

\section{Zhengwen Ye}

yezhengwen1300@163.com

1 Shanghai Key Laboratory of Protected Horticultural Technology, Forestry and Fruit Tree Research Institute, Shanghai Academy of Agricultural Sciences, Shanghai 201403, China

2 Horticultural Department, Shanghai Municipal Agricultural Technology Extension \& Service Center, Shanghai 201103, China 